\title{
Evaluation Of Mean Platelet Volume, Red Cell Distributed Width And Neutrophil To Lymphocyte Ratio In Conversion Disorder
}

This article was published in the following Dove Press journal:

Neuropsychiatric Disease and Treatment

\author{
Hasan Büyükaslan (D) \\ Mehmet Asoğlu² \\ 'Department of Emergency Medicine, \\ Harran University Medical Faculty, \\ Sanliurfa, Turkey; ${ }^{2}$ Psychiatry \\ Department, Harran University Medical \\ Faculty, Sanliurfa, Turkey
}

Background: The pathophysiology of conversion disorder (CD) is still not fully established. Many psychiatric disorders are known to be associated with inflammatory processes. We aimed to compare the routine hemogram values of $\mathrm{CD}$ patients with those of the participants in the healthy control group, to assess the inflammation levels of the two groups.

Methods: This study was conducted with CD patients $(n=158)$ and healthy controls $(n=145)$. Routine hematological parameters were examined in each participant. Group comparisons were made with Mann-Whitney U-test and Student's $t$-test. Regression analysis and receiver operating curve (ROC) analysis were also performed for the analysis of independent predictors.

Results: The comparisons revealed that while neutrophil-to-lymphocyte ratio (NLR), plateletto-lymphocyte ratio (PLR), platelet, neutrophil, red cell distribution width (RDW), and mean platelet volume (MPV) were significantly higher in CD group $(P<0.05)$, lymphocyte was decreased in that group $(P>0.05)$. Multivariate and ROC analyses showed MPV, RDW, and NLR as independent predictors $(P<0.05)$. ROC curve showed that MPV values of 7.8 or above could predict the CD with $84 \%$ sensitivity and $85 \%$ specificity (area under curve [AUC] $=0.878 ; 95 \%$ confidence interval $[\mathrm{CI}]: 0.817-0.939)$, RDW values of 11.0 or above could predict the $\mathrm{CD}$ with $82 \%$ sensitivity and $73 \%$ specificity (AUC $=0.871 ; 95 \% \mathrm{CI}$ : $0.815-0.926$ ), and NLR values of 1.8 or above could predict the $\mathrm{CD}$ with $85 \%$ sensitivity and $78 \%$ specificity (AUC $=0.865$; 95\% CI: 0.802-0.929).

Conclusion: $\mathrm{CD}$ is related to the acute inflammatory process. MPV, RDW, and NLR can reflect this inflammation. These parameters could be used in differential diagnosis; increased RDW and MPV levels can be used as a novel marker in CD.

Keywords: conversion disorder, inflammation, hematologic tests

\section{Introduction}

Conversion disorder (CD) is a common deficit and a psychiatric disorder that leads to several symptoms by affecting the motor and sensory functions. Some psychodynamic, neurobiological, genetic and socio-cultural factors are suggested in its etiology. ${ }^{1}$ In recent years, the effects of biological causes have been emphasized in its development. ${ }^{2}$ It is suggested that the symptoms of somatoform disorders are related to serotonergic neurotransmission. It is well known that serotonin regulates main functions related to pain and mood, and decreases in levels of serotonin metabolite 5-Hydroxy indol eacetic acid and tryptophan levels in somatoform disorders have been reported. . $^{3,4}$
Correspondence: Hasan Büyükaslan Department of Emergency Medicine, Harran University Medical Faculty, Sanliurfa, Turkey

Tel +905306446845

Email hasanbuyukaslan@hotmail.com 
Since the platelets express 5-hydroxytryptamine (HT) transporters and 5-HT 2A receptors, they are widely studied in psychiatry to understand their effects in serotonergic system. ${ }^{5} 5$-HT transporter is a mediator of 5-HT uptake from the plasma to platelets. Platelets can be used as markers to understand the mechanism of biochemical reactions in brain which occur in psychiatric diseases. ${ }^{6} \mathrm{~A}$ number of factors including psychological problems may have serious effects on platelets so they provide useful and rewarding data in order to understand the mechanisms in development of psychiatric disorders. ${ }^{6}$

The mean platelet volume (MPV) is widely used to assess the size and the function of the platelets. ${ }^{7}$ The megakaryocytes are large bone marrow cells which are in charge for the production, activation and function of platelets and the number of these cells are also used to determine the platelet volume. Under normal circumstances, the number and the size of the platelets have an inverse relationship. ${ }^{8,9}$ It has been suggested that MPV, platelet-to-lymphocyte ratio (PLR), red cell distribution width (RDW) and neutrophil-to-lymphocyte ratio (NLR) can be used to assess the inflammatory reactions occurring in various diseases. ${ }^{10}$ PLR and NLR are the most common markers of systemic inflammation. ${ }^{11}$ Furthermore, it has been shown that degradation of the erythrocytes or impaired erythropoiesis results in elevated RDW levels. ${ }^{12}$ The levels of RDW are also correlated with some markers of inflammation in a number of diseases. ${ }^{13,14}$

As far as we know, there are no reports investigating the association between the conversion disorder and the levels of blood components such as MPV, PLR, NLR and RDW. In this study, we intended to focus on this potential relationship in patients with $\mathrm{CD}$. It is hypothesized that CD patients will have more inflammatory reactions than individuals with no current or past functional neurological symptoms.

\section{Methods}

A total of 158 patients who were admitted to the emergency department of Harran University Hospital, Harran, Turkey, were recruited in this study. The local Ethics Committee of the institution approved the study and the study was performed according to the Declaration of Helsinki. An expert psychiatrist interviewed and examined all patients following the Diagnostic and Statistical Manual of Mental Disorders, 4th edition. ${ }^{15}$ The exclusion criteria were: Presence of depression, Parkinson's disease, multiple sclerosis, epilepsy, schizophrenia, migraine, anxiety, mental retardation, dementia and other psychotic and cognitive disorders. Patients who were users of alcohol, tobacco and pleasure-inducing substances were also excluded from the study. The control group consisted of volunteers non-clinical, general population who had no past psychiatric diagnosis or treatment. A mental interview was carried out to exclude mental or physical disorders. Drug, alcohol, tobacco and psychoactive substance users were excluded from the control group. They had no possible infectious disease, abnormal in the levels of the total aspartate aminotransferase (AST), alanine aminotransferase (ALT) or blood urea nitrogen (BUN). Each participant gave written informed consent before the study.

\section{Collection And Preparation Of Blood Samples}

The blood of the patients and the controls was drawn and collected in $3 \mathrm{~mL}$ ethylenediaminetetraacetic acid (EDTA) tubes. Complete blood count (CBC) including RDW, MPV, mean corpuscular volume (MCV), white blood cell (WBC), hemoglobin, lymphocyte, neutrophil and platelet counts was performed on the first day of admission to the emergency department. The PLR and the NLR were taken into account. The $\mathrm{CBC}$ was performed using an automated analyzer (Cell-Dyn Ruby 3200, Abbott Laboratories, Santa Clara, CA, USA).

\section{Statistical Analysis}

All parameters were calculated using SPSS 20 (IBM Corporation, Armonk, NY, USA) including the mean and the standard deviation. The normality of the data was calculated using the Kolmogorov-Smirnov test. MannWhitney $U$-test was used to compare the variables such as hemoglobin, lymphocytes, neutrophils, MPV, PLR, RDW and NLR. The normally distributed variables of the patients were compared using Student's $t$-test. Additionally, the categorical variables between groups were calculated using chi-square test. To estimate the presence of $\mathrm{CD}$, logistic regression analyses (univariate and stepwise multivariate) and receiver operating curve analyses (ROC) were performed. $\mathrm{p}<0.05$ was accepted as significant difference.

\section{Results}

Our study included 158 patients with CD and 145 healthy control subjects. The laboratory results and the demographic characteristics of the participants are presented in Table 1. Age, gender and the place of residence were 
Table I Demographic And Laboratory Characteristics Of Two Groups

\begin{tabular}{|c|c|c|c|}
\hline Variables & CD Group $(n=158)$ & Control Group $(n=145)$ & $P$ value \\
\hline Age (years) & $34.4(30.8-38.2)$ & $35.9(32.0-40.1)$ & $0.439 *$ \\
\hline Gender (Male) & $86(54.4)$ & 77 (53.4) & 1.000 \\
\hline Urban & $102(64.7)$ & $84(53.4)$ & 0.174 \\
\hline Symptoms & & - & \\
\hline Attacks or seizures & $95(60.0 \%)$ & - & \\
\hline Anaesthesia or sensory loss & $47(30.0 \%)$ & - & \\
\hline Weakness or paralysis & $8(5.0 \%)$ & - & \\
\hline Speech symptoms & $8(5.0 \%)$ & & \\
\hline WBC $\left(10^{3} / \mu \mathrm{L}\right)$ & $8.4(8.0-8.9)$ & $7.7(7.3-8.9)$ & 0.074 \\
\hline Neutrophil $(103 / \mu \mathrm{L})$ & $5.4(5.0-5.9)$ & $3.9(3.7-4.2)$ & $<0.001 * *$ \\
\hline Lymphocyte $(103 / \mu \mathrm{L})$ & $2.2(2.1-2.4)$ & $2.4(2.3-2.5)$ & $0.001 *$ \\
\hline Hemoglobin $(g / d L)$ & $13.9(\mid 3.5-14.3)$ & $13.8(|3.4-| 4.1)$ & 0.852 \\
\hline $\mathrm{MCV}(\mathrm{fL})$ & $85.7(84.4-86.9)$ & $86.3(85.1-87.5)$ & 0.602 \\
\hline MPV (fL) & $8.8(8.5-9.0)$ & $7.5(7.3-7.6)$ & $<0.001 * *$ \\
\hline Platelet $(103 / \mu \mathrm{L})$ & $283.7(264.6-305.2)$ & $267.3(253.8-282.1)$ & $0.021 *$ \\
\hline RDW (fL) & $12.3(12.0-12.8)$ & $10.6(10.3-10.9)$ & $<0.001 * *$ \\
\hline NLR & $2.7(2.4-3.1)$ & $1.7(1.6-1.8)$ & $<0.001 * *$ \\
\hline PLR & $138.9(\mid 26.1-153.4)$ & $116.1(107.3-126.6)$ & $<0.001 * *$ \\
\hline
\end{tabular}

Notes: Values were presented as mean (Confidence Interval) or number (\%). *P values were obtained from the Mann-Whitney U-test. Bold values indicate significance. $* p<0.05 ; * *_{p}<0.001$.

Abbreviations: WBC, white blood cell; MCV, mean corpuscular volume; MPV, mean platelet volume; RDW, red distributed width; NLR, neutrophil to lymphocyte ratio; PLR, platelet to lymphocyte ratio.

similar in both groups. While RDW, NLR, MPV, PLR, neutrophil and platelet levels were significantly high, the lymphocyte count was significantly low in patient group when compared to the control group $(\mathrm{p}<0.05)$ (Figure 1).

The univariate logistic regression analyses revealed that MPV, NLR and RDW could be used as predictors of CD. In addition, the stepwise multivariate logistic regression analyses demonstrated that NLR, MPV and RDW were independent predictors for the estimation of the presence of $\mathrm{CD}$ in the final model (Table 2). Similarly, ROC analyses also demonstrated that MPV values of 7.8 or above could predict the CD with $84 \%$ sensitivity and $85 \%$ specificity (area

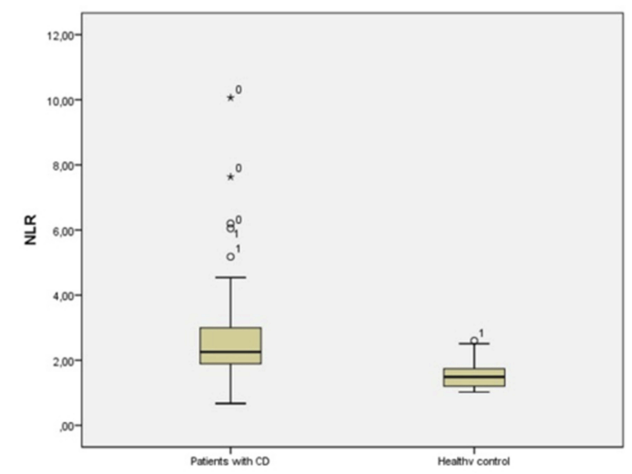

under curve (AUC) $=0.878$; 95\% CI: 0.817-0.939), RDW values of 11.0 or above could predict the $\mathrm{CD}$ with $82 \%$ sensitivity and $73 \%$ specificity (AUC $=0.871 ; 95 \%$ CI: $0.815-0.926$ ) and NLR values of 1.8 or above could predict the $\mathrm{CD}$ with $85 \%$ sensitivity and $78 \%$ specificity (AUC $=0.865$; 95\% CI: 0.802-0.929) (Figure 2).

\section{Discussion}

This study is the first to demonstrate hematologic inflammatory markers such as RDW, NLR, MPV and PLR levels in CD patients. Patients with CD had significantly higher RDW, NLR, MPV, PLR, neutrophil and platelet counts

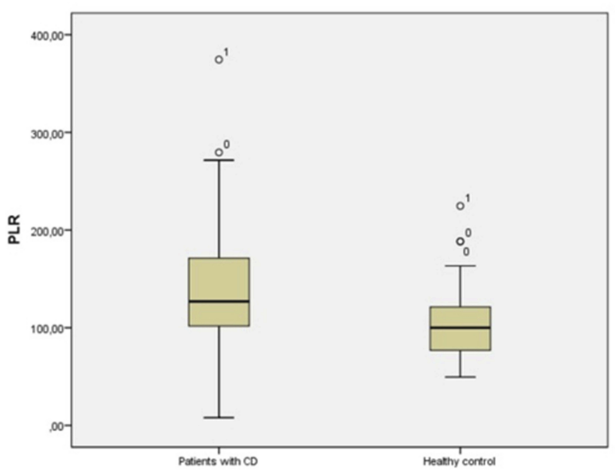

Figure I The Boxplot graph demonstrating the statistical differences of NLR and PLR between groups.

Notes: *Symbol indicates end stage limits of patients populations in Figure I.

Abbreviations: NLR, neutrophil-to-lymphocyte ratio; PLR, platelet-to-lymphocyte ratio. 
Table 2 Univariate And Multivariate Analyses For The Estimation Of Conversion Disorder Presence

\begin{tabular}{|l|l|l|l|l|l|l|}
\hline \multirow{2}{*}{ Variables } & \multicolumn{2}{l|}{ Univariate Analyses } & \multicolumn{2}{l|}{ Multivariate Analyses (Stepwise) } \\
\cline { 2 - 7 } & P value & Odss Ratio & $\mathbf{9 5 \%} \mathbf{~ C l}$ & P value & Odds Ratio & $\mathbf{9 5 \%}$ Cl \\
\hline NLR & $<0.001$ & 0.066 & $0.025-0.177$ & 0.003 & 0.106 & $0.024-0.459$ \\
PLR & $<0.001$ & 0.983 & $0.974-0.992$ & $>0.05$ & & \\
RDW & $<0.001$ & 0.265 & $0.170-0.411$ & $<0.001$ & 0.219 & $0.108-0.444$ \\
MPV & $<0.001$ & 0.169 & $0.095-0.301$ & $<0.001$ & 0.148 & $0.060-0.366$ \\
\hline
\end{tabular}

Abbreviations: $\mathrm{Cl}$, confidence interval; NLR, neutrophil to lymphocyte ratio; PLR, platelet to lymphocyte ratio; RDW, red distributed width; MPV, mean platelet volume.

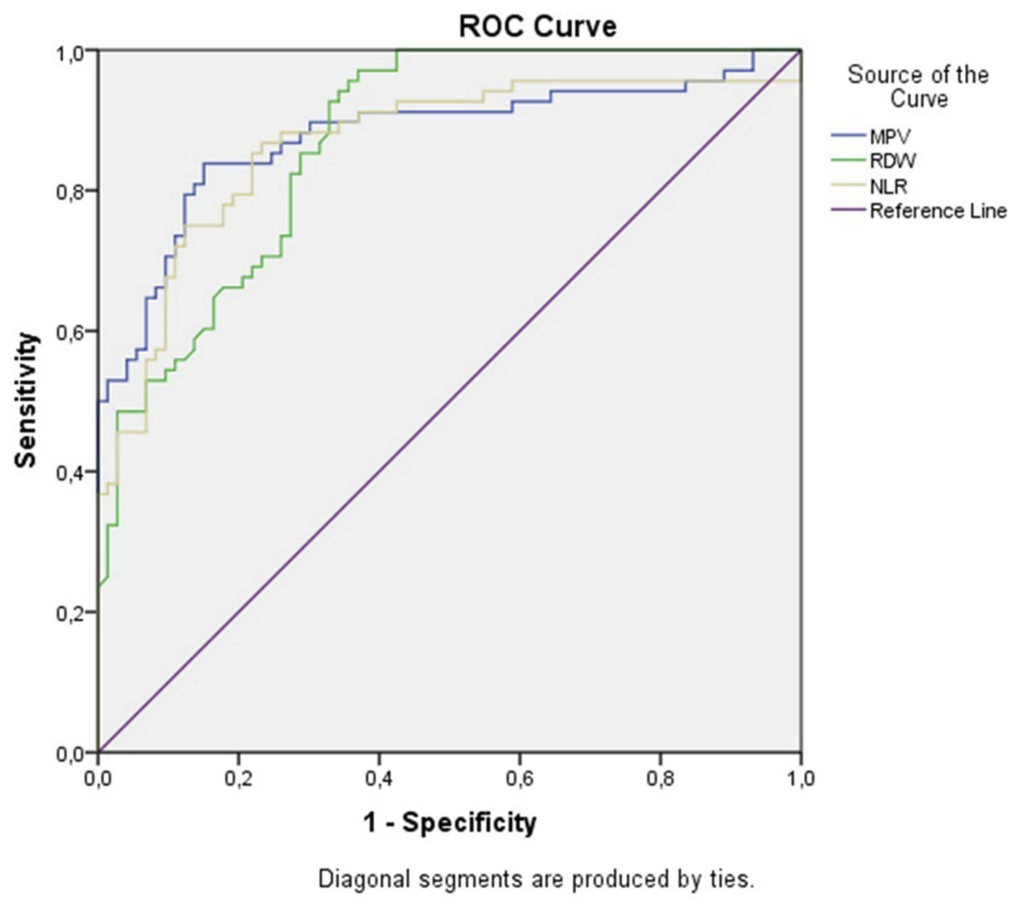

Figure 2 ROC curve analyses for the estimation of conversion disorder presence. Receiver operating characteristic (ROC) analysis for the presence of conversion disorder (CD). ROC curve showed that MPV values of 7.8 or above could predict the CD with $84 \%$ sensitivity and $85 \%$ specificity (area under curve (AUC) $=0.878$; $95 \%$ confidence interval (Cl): $0.817-0.939)$, RDW values of II.0 or above could predict the CD with $82 \%$ sensitivity and $73 \%$ specificity (AUC=0.87I; $95 \%$ CI: 0.8 I $5-0.926)$ and NLR values of 1.8 or above could predict the CD with $85 \%$ sensitivity and $78 \%$ specificity (AUC=0.865; $95 \% \mathrm{Cl}: 0.802-0.929$ ).

Abbreviations: MPV, mean platelet volume RDW, red distributed width; NLR, neutrophil to lymphocyte ratio.

than healthy controls, while lymphocyte count was significantly lower and NLR, MPV and RDW were important predictors in $\mathrm{CD}$.

Human body gives inflammatory reactions after cellular damage, infection or stress which are all considered necessary and appropriate. ${ }^{16}$ On the other hand, these reactions can be damaging and inappropriate in case of wrong or excess stimuli which can result in energy, sleep, motivation and cognitive disorders. ${ }^{17}$ The pathophysiology of conversion disorder is complex and the role of systemic inflammation is not clarified yet. A couple of studies have reported the extensive role of peripheral immune modulators in psychiatric disorders. ${ }^{16,18}$
It has been shown that the injection of interleukin-1 and tumor necrosis factor alpha as pro- inflammatory cytokines to healthy animals lead to disorders of loneliness behaviors. ${ }^{10}$ Some other immunological or inflammatory diseases such as rheumatoid arthritis, multiple sclerosis, obesity and diabetes act as risk factors for the psychiatric disease and it has been suggested that prevalent inherent inflammatory processes influence the brain. ${ }^{10,16,18-21}$

Signals related to the immune system are transmitted to the brain through several ways. ${ }^{10}$ Cytokines have significant effects on the serotonin production mechanism in the brain. Furthermore, a body of evidence has suggested that these cytokines activate hypothalamic- pituitary-adrenal 
axis leading to induction of glucocorticoid insensitivity. ${ }^{22}$ We demonstrated that MPV, NLR and RDW can be used as the independent predictors of CD as these are cheap and repeatable inflammatory markers which can effectively show the relationship between inflammation and psychiatric disease. Some of the studies have demonstrated that there is a blood-brain relationship between platelets and the neuronal 5-HT transporter. ${ }^{6}$ There is a correlation between platelets and synaptosomal re-uptake. Some changes can be found in the human blood, brain and cerebrospinal fluid 5-HT levels, following the administration of 5-HT-releasing agents. Therefore, we conclude that an abnormal 5-HT metabolism in platelets may indicate the abnormal function of platelets and increased MPV levels. We determined that blood component values on CBC (platelet count, RDW, NLR and MPV) were statistically different between the groups of $\mathrm{CD}$ and controls.

It has been shown that alteration in serum inflammatory markers can be detected during depressive and manic periods, and also in patients with major depression and anxiety. ${ }^{23-25}$ Several studies indicate that the levels of PLR and NLR were increased in various neuropsychiatric disorders. ${ }^{16,17,26}$ Furthermore, increased MPV values as an inflammatory marker have been shown in previous studies focusing on major depression, schizophrenia, and panic, bipolar and generalized anxiety disorders. ${ }^{10,19-21}$ In many studies, it has been shown that RDW, a marker of heterogeneity in erythrocyte size, is associated with inflammatory parameters. ${ }^{13,14}$ Increased RDW values have been detected in patients with panic disorder and major depression. ${ }^{27,28}$ In our study, the levels of PLR, NLR, RDW and MPV were higher than the controls in patients with $\mathrm{CD}$; then again, NLR, MPV and RDW were independent predictors of the $\mathrm{CD}$.

Our results supported that inflammatory process is activated during attack period in patients with CD. Moreover, these parameters can easily and effectively be used as cheap, simple and repeatable parameters of routine blood count test in diagnosis of CD.

Our control group which consisted of healthy volunteers can be considered as the major limitation of our study. Therefore, we could not assess the significance of MPV, RDW and NLR in differential diagnosis of CD with another disease including neurologic and psychiatric disorders and malingering.

\section{Conclusion}

$\mathrm{CD}$ may be related to the acute inflammatory process. The components of blood count test such as MPV, NLR and
RDW can reflect the inflammation and these parameters can be used in differential diagnosis. Thus, increased RDW and MPV levels can be used as a novel marker in CD. However, further and comprehensive studies are necessary to support the present findings.

\section{Disclosure}

The authors report no conflicts of interest in this work.

\section{References}

1. Ford CV, Folks DG. Conversion disorders: an overview. Psychosomatics. 1985;26:371-383. doi:10.1016/S0033-3182(85)72845-9

2. Büyükaslan H, Kandemir SB, Asoğlu M, et al. ŞavikE. Evaluation of oxidant, antioxidant, and S100B levels in patients with conversion disorder. Neuropsychiatr DisTreat. 2016;12:1725-1729.

3. Rief W, Pilger F, Ihle D, Verkerk R, Scharpe S, Maes M. Psychobiological aspects of somatoform disorders: contributions of monoaminergic transmitter systems. Neuropsychobiology. 2004;49 (1):24-29. doi:10.1159/000075335

4. Anderson G, Maes M, Berk M. Biological underpinnings of the commonalities in depression, somatization, and chronic fatigue syndrome. Med Hypotheses. 2012;78(6):752-756. doi:10.1016/j.mehy.2012.02.023

5. Young JJ, Bruno D, Pomara N. A review of the relationship between proinflammatory cytokines and major depressive disorder. $J$ Affect Disord. 2014;169:15-20. doi:10.1016/j.jad.2014.07.032

6. Camacho A, Dimsdale JE. Platelets and psychiatry: lessons learned from old and new studies. Psychosom Med. 2000;62(3):326-336. doi:10.1097/00006842-200005000-00006

7. GogcegozGul I, Eryılmaz G, Ozten E, HızlıSayar G. Decreased mean platelet volume in panic disorder. Neuropsychiatr Dis Treat. 2014; 10:1665-1669.

8. Threatte GA. Usefulness of the mean platelet volume. Clin Lab Med. 1993;13(4):937-950.

9. Boyraz I, Koç B, Boyacı A, Tutoğlu A, Sarman H, Ozkan H. Ratio of neutrophil/lymphocyte and platelet/lymphocyte in patient with ankylosing spondylitis that are treating with anti-TNF. Int J ClinExp Med. 2014;7(9):2912-2915.

10. Kilincalp S, Coban S, Akinci H, et al. Neutrophil/lymphocyte ratio, platelet/lymphocyte ratio, and mean platelet volume as potential biomarkers for early detection and monitoring of colorectal adenocarcinoma. Eur J Cancer Prev. 2015;24(4):328-333. doi:10.1097/ CEJ.0000000000000092

11. Balta S, Demirkol S, Kucuk U. The platelet lymphocyte ratio may be useful inflammatory indicator in clinical practice. HemodialInt. 2013;17(4):668-669.

12. Bessman JD. More on the RDW. Am J Clin Pathol. 1985;84(6):773.

13. Duchnowski P, Szymański P, Orłowska-Baranowska E, Kuśmierczyk M, Hryniewiecki T. Raised red cell distribution width as a prognostic marker in aortic valve replacement surgery. Kardiol Pol. 2016;74 (6):547-552.

14. Altiparmak IH, Erkus ME, Kocarslan A, et al. High aortic pulse-wave velocity may be responsible for elevated red blood cell distribution width in overweight and obese people: a community-based, crosssectional study. Cardiovasc J Afr. 2016;27:1. doi:10.5830/CVJA2016-005

15. American Psychiatric Association. Diagnostic and Statistical Manual of Mental Disorders (DSM-IV-TR). Washington, D.C.: American Psychiatric Association; 2000.

16. Mert DG, Terzi H. Mean platelet volume in bipolar disorder: the search for an ideal biomarker. Neuropsychiatr Dis Treat. 2016;Aug16 (12):2057-2062. doi:10.2147/NDT.S112374 
17. Kalelioglu T, Akkus M, Karamustafalioglu N, et al. Neutrophil-lymphocyte and platelet- lymphocyte ratios as inflammation markers for bipolar disorder. Psychiatry Res. 2015;228(3):925-927. doi:10.1016/ j.psychres.2015.05.110

18. Topal E, Celiksoy MH, Catal F, Karakoç HT, Karadağ A, Sancak R. The platelet parameters as inflammatory markers in preschool children with atopic eczema. Clin Lab. 2015;61(5-6):493-496.

19. Kayhan F, Gündüz Ş, Ersoy SA, Kandeğer A, Annagür BB. Relationships of neutrophil- lymphocyte and platelet-lymphocyte ratios with the severity of majör depression. Psychiatry Res. 2017;247:332-335. doi:10.1016/j.psychres.2016.11.016

20. Almis BH, Aksoy I. Mean platelet volume level in patients with generalized anxiety disorder. Psychiatry Clin Psychopharmacol. 2018;28(1):43-47. doi:10.1080/24750573.2017.1385210

21. Wysokinski A, Szczepocka E. Platelet parameters (PLT, MPV, P-LCR) in patients with schizophrenia, unipolar depression and bipolar disorder. Psychiatry Res. 2016;237:238-245. doi:10.1016/j.psychres.2016.01.034

22. Alcaíno $\mathrm{H}$, Chiong $\mathrm{M}$, Reyes $\mathrm{D}$, et al. Red cell distribution width as biomarker in older adults: a brief review. MOJ Gerontol Ger. 2017;2 (3):61-62.
23. Remlinger-Molenda A, Wójciak P, Michalak M, et al. Activity of selected cytokines in bipolar patients during manic and depressive episodes. Psychiatry Pol. 2012;46(4):599-611.

24. Vogelzangs N, Beekman AT, de Jonge P, Penninx BW. Anxiety disorders and inflammation in a large adult cohort. Transl Psychiatry. 2013. doi:10.1038/tp.2013.27

25. Fond G. Inflammation in psychiatric disorders. European Psychiatry. 2014;29:551-552. doi:10.1016/j.eurpsy.2014.09.347

26. Kuyumcu ME, Yesil Y, Ozturk ZA, et al. The evaluation of neutrophil lymphocyte ratio in Alzheimer's disease. Dement Geriatr Cogn Disord. 2012;34(2):69-74.

27. Demircan F, Gözel N, Kılınç F, Ulu R, Atmaca M. The impact of red blood cell distribution width and neutrophil/lymphocyte ratio on the diagnosis of major depressive disorder. Neurol Ther. 2016;5(1):2733. doi:10.1007/s40120-015-0039-8

28. Asoglu M, Aslan M, Imre O, et al. Mean platelet volume and red cell distribution width levels initial evaluation of panic disorder. Neuropsychiatr Dis Treat. 2016;22(12):2435-2438. doi:10.2147/ NDT.S111108
Neuropsychiatric Disease and Treatment

\section{Publish your work in this journal}

Neuropsychiatric Disease and Treatment is an international, peerreviewed journal of clinical therapeutics and pharmacology focusing on concise rapid reporting of clinical or pre-clinical studies on a range of neuropsychiatric and neurological disorders. This journal is indexed on PubMed Central, the 'PsycINFO' database and CAS, and

\section{Dovepress}

is the official journal of The International Neuropsychiatric Association (INA). The manuscript management system is completely online and includes a very quick and fair peer-review system, which is all easy to use. Visit http://www.dovepress.com/testimonials.php to read real quotes from published authors. 